\title{
Value of monitoring urine ammonia at time of biopsy in patients with lupus nephritis
}

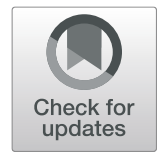

Huanhuan Zhu', Huiting Wan ${ }^{\dagger}$, Suyan Duan ${ }^{\dagger}$, Chengning Zhang, Qing Li, Simeng Liu, Lin Wu, Bo Zhang, Changying Xing $^{\dagger}$ and Yanggang Yuan ${ }^{*+}$

\begin{abstract}
Objective: Although lupus nephritis (LN) is mostly characterized by glomerular involvement, tubular injury is indispensable in its pathogenesis and progression. The purpose of this study is to examine associations between urinary acidification function and clinical and pathological features in LN.

Methods: A total of 103 patients with renal biopsy-proven LN were included, and clinical parameters and laboratory data were obtained from the medical records. Plasma samples, 24-h urine samples and the urinary acidification function, including urine $\mathrm{pH}$, titratable acid, and ammonia, were collected within 3 days before the day of renal biopsy. The correlations between defects of acid excretion and clinical and pathological features were then assessed. Logistic regression analysis was used to assess factors associated with the presence of nephrotic range proteinuria.

Results: The urine ammonia level was inversely correlated with SLEDAI-2 K scores, rSLEDAI scores, serum creatinine levels and proteinuria, while it was positively correlated with eGFR. And urine titratable acid was only inversely correlated with rSLEDAI scores and proteinuria. Moreover, urine ammonia had significant negative correlations with Al scores, interstitial inflammatory cell infiltration, Cl scores, glomerular sclerosis, fibrous crescents, tubular atrophy and interstitial fibrosis. And urine titratable acid was mainly inversely correlated with $\mathrm{Cl}$ scores. Furthermore, univariate logistic analyses identified that both urine titratable acid and ammonia were correlated with the presence of nephrotic range proteinuria. After the adjustment for chronicity index and eGFR in a multivariate logistic analysis, only urine titratable acid was still identified as an independent risk factor for the occurrence of nephrotic range proteinuria.

Conclusions: Urine ammonia was associated with clinical and pathological features of chronicity and tubulointerstitial disease activity among patients with lupus nephritis. Furthermore, the strong association between urinary protein and titratable acid excretion at the time of kidney biopsy is significant even after adjusting for the chronicity index and eGFR at biopsy.
\end{abstract}

Keywords: Lupus nephritis, Urinary acidification function, Urine ammonia, Tubulointerstitial lesions

\footnotetext{
* Correspondence: ygyuan@njmu.edu.cn

Huanhuan Zhu, Huiting Wan and Suyan Duan are Co-first authors of this study.

${ }^{\dagger}$ Changying Xing and Yanggang Yuan are senior authors with the equal contribution in this study.

Department of Nephrology, The First Affiliated Hospital of Nanjing Medical University, Nanjing Medical University, 300 Guangzhou Road, Nanjing, Jiangsu Province 210029, P.R. China
} 


\section{Background}

Lupus nephritis (LN) carries high morbidity and mortality in patients with systemic lupus erythematosus (SLE), and $40 \%$ of patients with SLE will develop renal impairment for different degrees $[1,2]$. The risk of end-stage kidney disease (ESRD) is high in lupus nephritis (up to 10\%) [3]. Thus, accurate indicators are urgently needed to reflect the renal activity of LN and identify patients at high risks of progression to ESRD.

Unfortunately, conventional biomarkers such as serum creatinine levels, anti-dsDNA antibody titers, and complement levels perform poorly in predicting lupus renal flares and prognostic stratification [4]. Renal biopsy, the gold standard for diagnosis, represents the nature and severity of renal involvement to evaluate the risk of renal failure [5]. As an invasive diagnostic test, a repeat renal biopsy is unrealistic for longitudinally monitoring the response to therapy or the activity of lupus nephritis. Urinary biomarkers offer a window for assessing the response to treatment and the impending renal flare, which are excreted directly from the kidney and able to longitudinal monitoring. Proteinuria as the major candidate of urine presents a weak connection with the historical activity in LN and adverse renal outcomes [6]. There are several novel biomarkers including urinary angiostatin, vascular cell adhesion molecule 1, immunoglobulin binding protein 1 , and the TNF-like weak inducer of apoptosis, which have been examined to reflect histological features of lupus nephritis and identify higher risk for renal outcome [710]. However, these biomarkers are still in its infancy and have not yet been widely used in clinical settings.

Renal tubular acidosis (RTA) is a defect of tubular function, which is characterized by the renal inability to reabsorb enough bicarbonate $\left(\mathrm{HCO}_{3}{ }^{-}\right)$to a proximal tubule or to secrete enough hydrogen $\left(\mathrm{H}^{+}\right)$through a distal tubule $[11,12]$. The hydrogen is secreted into the final urine by the excretion of titratable acid and ammonia. There is an intimate connection between RTA and kidney disease such as diabetic nephropathy, tubulointerstitial nephropathies, and autoimmune disorder [13]. It is of note that renal acidification defects are associated with the risk of chronic kidney disease (CKD) progression. $M$ Vallet et al. in the NephroTest cohort with CKD found urinary ammonia excretion decreased with the elevated glomerular filtration rate (GFR), which was associated with a significantly higher risk of kidney disease progression [14]. In diabetic nephropathy, patients exhibited a lower risk of progression toward ESRD in higher net acid excretion [15]. This is consistent with our previous study, which showed that defects of titratable acid and ammonia secretion were associated with GFR and the dysfunction of titratable acid secretion was an independent predictor for diabetic nephropathy progression [16]. It has been known that tubular lesions were common in lupus nephritis patients and correlated closely with renal outcomes [3, 17, 18]. In addition, RTA is a complication of lupus nephritis with a variety of tubular dysfunction and is intimately associated with disease activities [19].

Previous work has focused primarily on types of RTA and laboratory indices in LN. The role of urinary acidification function rather than overt metabolic acidosis in LN remains unknown. Therefore, the purpose of this study was to examine the association between defects of acid excretion and clinical and pathological features in LN.

\section{Methods \\ Patients}

A total of 103 patients with renal biopsy-proven lupus nephritis were recruited by the First Affiliated Hospital of Nanjing Medical University from January 2003 to October 2018 in this study. The inclusion criteria were as follows: (1) patients $\geq 18$ years of age; (2) these fulfilled the 2012 SLE international collaborating clinics classification criteria [20]; (3) these with biopsy-proven lupus nephritis according to the International Society of Nephrology/Renal Pathology Society (ISN/RPS) in 2003 classification system [21]. And the exclusion criteria were as follows: (1) patients with other kidney diseases (such as minimal change disease and thrombotic microangiopathy) or diabetes; (2) these who took some sodium bicarbonate tablets or diuretics within 30 days. The ethical committee of the First Affiliated Hospital of Nanjing Medical University approved this study based on the Declaration of Helsinki. All participants signed written informed consent.

\section{Measurements}

Clinical parameters and laboratory data were obtained from the medical records, including gender, age, SLE duration, blood pressure, serum creatinine, blood urea nitrogen (BUN), cystatin C, albumin, complement 3 (C3), antinuclear antibodies (ANA), anti-double-stranded DNA (antidsDNA), 24-h urine protein and so on. Plasma samples and 24-h urine samples were obtained from patients within 3 days before renal biopsy and before any immunosuppressive treatment. GFR was estimated using the chronic kidney disease epidemiology collaboration (CKD-EPI) equation [22]. The SLE disease activity index 2000 (SLEDAI-2 K) and renal SLE disease activity index (rSLEDAI) were used to assess SLE activity and kidney disease activity, respectively $[7,23]$. The rSLEDAI consists of four parameters: hematuria, proteinuria, pyuria and urinary casts, each accounting for four scores. The evaluation of SLEDAI-2 K scores, rSLEDAI scores and the diagnosis of nephrotic syndrome were obtained 1 day before renal biopsy.

After 3 days of vegetarian diets, the urinary acidification function was detected from a fasting morning urine sample within 3 days before the day of renal biopsy, which 
was measured using a ZDJ-4B automatic potentiometric titrator (Shanghai INESA Scientific Instrument Co., Shanghai, China), as described in our previous study [16]. Normal values of the urinary acidification function are as follows: urine $\mathrm{pH}$ (5.0--8.0), titratable acid $\geq 10 \mathrm{mmol} / \mathrm{L}$ and ammonia $\geq 20 \mathrm{mmol} / \mathrm{L}$.

\section{Renal histology}

Renal biopsy specimens were examined by two experienced pathologists following the 2003 ISN/RPS classification system [21]. The activity index (AI) and chronicity index (CI) of lupus nephritis were assessed using the semiquantitative scoring of the National Institutes of Health $[24,25]$. Activity indices consisted of endocapillary proliferation, cellular crescents, fibrinoid necrosis, subendothelial hyaline deposits, interstitial inflammatory cell infiltration, and glomerular leukocyte infiltration. Chronicity indices consisted of glomerular sclerosis, fibrous crescents, tubular atrophy, and interstitial fibrosis. The segmental changes of indices were scored as follows: 0 , normal; $1,<25 \%$ of the acreage; $2,25-50 \%$ of the acreage; and 3, $>50 \%$ of the acreage in each specimen. And glomerular sclerosis, cellular crescents, and fibrous crescents were calculated as percentages of the total number of glomeruli. The fibrinoid necrosis and cellular crescents were weighted by a factor of 2 .

\section{Statistical analysis}

All statistical analyses were evaluated using SPSS version 22 (SPSS, Chicago, IL, USA). Variables were presented as mean \pm standard deviation, median (interquartile range), median with range (minimum, maximum) or percentage. Student's t-test and one-way analysis of variance were used for data with a normal distribution. Mann-Whitney $\mathrm{U}$ test and Kruskal-Wallis test were used for data with a non-normal distribution. And the Chi-square test was used for qualitative variables. Spearman's rank correlation was performed for correlation analysis of various lesions. Logistic analysis was performed to evaluate the association of urinary acidification function with nephrotic range proteinuria ( $24 \mathrm{~h}$ urinary protein $>3.5 \mathrm{~g}$ ). The variables that showed a statistically significant association in univariate analysis were adjusted in a multivariate model. Results were expressed as odds ratios (OR) with 95\% confidence intervals $(95 \% \mathrm{CI})$. Statistical significance was considered as $p<0.05$.

\section{Results}

Baseline characteristics of lupus nephritis

A total of 103 patients with lupus nephritis were included in this study. The clinical characteristics, laboratory parameters and pathological features of patients were shown in Table 1 . The majority (83.5\%) of the patients were female. The mean age was $39.75 \pm 14.39$ years, and the mean duration of SLE was $4(1,24)$ months. The mean levels of eGFR, proteinuria, serum creatinine and C3 were 86.76 $(52.11,117.36) \mathrm{ml} / \mathrm{min} / 1.73 \mathrm{~m}^{2}, \quad 2.94(1.29,5.51) \mathrm{g} / 24 \mathrm{~h}$, $76.30(53.70,116.20) \mathrm{umol} / \mathrm{L}$ and $0.49(0.37,0.82) \mathrm{g} / \mathrm{L}$, respectively. According to the ISN/RPS 2003 classification system, patient distribution by the pathological stages was as follows: class III, 14 (13.59\%), class IV, 60 (58.25\%) and

Table 1 Baseline characteristics in patients with lupus nephritis $(n=103)$

\begin{tabular}{|c|c|c|c|c|c|}
\hline \multicolumn{2}{|l|}{ Clinical evaluation } & \multicolumn{2}{|l|}{ Laboratory assessment } & \multicolumn{2}{|c|}{ Renal histopathology indices (median, range) } \\
\hline Female,n(\%) & $86(83.50)$ & WBC(10^9/L) & $4.73(3.50,7.06)$ & Al scores & $4(1,10)$ \\
\hline Age (years) & $39.75 \pm 14.39$ & $\mathrm{Hb}(\mathrm{g} / \mathrm{L})$ & $100(90,116)$ & Endocapillary hypercellularity & $0(0,1)$ \\
\hline SLE duration (months) & $4(1,24)$ & $\operatorname{PLT}(10 \wedge 9 / L)$ & $167.50 \pm 67.34$ & Cellular crescents & $0(0,6)$ \\
\hline Hypertension,n (\%) & $35(33.98)$ & $\mathrm{eGFR}\left(\mathrm{ml} / \mathrm{min} / 1.73 \mathrm{~m}^{2}\right)$ & $86.76(52.11,117.36)$ & Karyorrhexis/fibrinoid necrosis & $0(0,2)$ \\
\hline Hematuria,n(\%) & $54(52.43)$ & Urine protein(g/24h) & $2.94(1.29,5.51)$ & Subendothelial hyaline deposits & $1(0,2)$ \\
\hline Nephrotic syndrome,n(\%) & $42(40.78)$ & Cystatin C (mg/L) & $1.57(1.09,2.35)$ & Interstitial inflammatory cell infiltration & $2(1,3)$ \\
\hline SLEDAI-2 K & $11(9,24)$ & $\mathrm{SCr}(\mathrm{umol} / \mathrm{L})$ & $76.30(53.70,116.20)$ & Glomerular leukocyte infiltration & $0(0,1)$ \\
\hline rSLEDAI & $4(4,8)$ & $\mathrm{BUN}(\mathrm{mmol} / \mathrm{L})$ & $6.93(4.67,11.10)$ & Cl scores & $2(0,11)$ \\
\hline $\mathrm{SBP}(\mathrm{mmHg})$ & $130(122,143)$ & Uric acid (umol/L) & $407.14 \pm 125.09$ & Glomerular sclerosis & $1(0,3)$ \\
\hline \multirow[t]{7}{*}{$\mathrm{DBP}(\mathrm{mmHg})$} & $85(78,91)$ & Albumin(g/L) & $25.72 \pm 7.39$ & Fibrous crescents & $0(0,3)$ \\
\hline & & Anti-ANA antibodies (+), n (\%) & $96(93.20)$ & Tubular atrophy & $1(0,3)$ \\
\hline & & Anti-Sm antibodies(+), n (\%) & $54(52.43)$ & Interstitial fibrosis & $0(0,3)$ \\
\hline & & Anti-dsDNA antibodies(+), n (\%) & $63(61.17)$ & & \\
\hline & & $\lg G(g / L)$ & $12.75(7.56,16.15)$ & & \\
\hline & & $\mathrm{C} 3(\mathrm{~g} / \mathrm{L})$ & $0.49(0.37,0.82)$ & & \\
\hline & & $\mathrm{C} 4(\mathrm{~g} / \mathrm{L})$ & $0.10(0.07,0.17)$ & & \\
\hline
\end{tabular}

Values for categorical data were given as a number (percent); values for continuous variables were expressed as mean \pm standard deviation (normally distributed data) or median (interquartile range) (non-normally distributed data); values for renal histopathology indices were expressed as median (minimum, maximum) 
class V, 29 (28.16\%). The median levels of the activity indices and the chronicity indices were $4(1,10)$ and $2(0,11)$, respectively.

\section{Associations between urinary acidification function and clinical characteristics of lupus nephritis}

Patients of lupus nephritis with nephrotic syndrome or nephrotic range proteinuria $(>3.5 \mathrm{~g} / 24 \mathrm{~h}$ ) had higher urine $\mathrm{pH}$ levels and lower urine titratable acid excretion. The level of urine ammonia was lower in patients with nephrotic range proteinuria. Urine ammonia excretion was less in males, whereas urine $\mathrm{pH}$ and titratable acid excretion did not differ between the sexes in lupus nephritis. There were no statistically significant differences in groups concerning age, hypertension, hematuria, and hypokalemia (Table 2).

Urine $\mathrm{pH}$ was positively correlated with rSLEDAI scores, eGFR, and proteinuria, whereas it was inversely correlated with serum creatinine levels. There were significantly negative correlations between urine titratable acid and rSLEDAI scores and proteinuria. Urine ammonia was inversely correlated with SLEDAI- $2 \mathrm{~K}$ scores, rSLEDAI scores, serum creatinine levels, and proteinuria, while it was positively correlated with eGFR. There were no other differences between urinary acidification function and other clinical characteristics.

Moreover, univariate logistic regression analyses identified that both urine titratable acid and ammonia were correlated with the presence of nephrotic range proteinuria. After the adjustment for chronicity index and eGFR in a multivariate logistic regression analysis, urine titratable acid was still identified as an independent risk factor for the occurrence of nephrotic range proteinuria in patients with lupus nephritis. However, urine ammonia showed no association after the multivariable adjustment (Fig. 1).

\section{Associations between urinary acidification function and renal pathological features of lupus nephritis}

As shown in Table 2, urinary acidification function did not associate with endocapillary hypercellularity, cellular crescents, karyorrhexis/fibrinoid necrosis or subendothelial hyaline deposits. Urine $\mathrm{pH}$ was positively correlated with tubular atrophy. There was significantly positive correlation between urine titratable acid and glomerular leukocyte infiltration, whereas urine titratable acid was negatively correlated with CI scores, glomerular sclerosis, tubular atrophy, and interstitial fibrosis. Urine ammonia had significant negative correlations with AI scores, interstitial inflammatory cell infiltration, CI scores, glomerular sclerosis, fibrous crescents, tubular atrophy and interstitial fibrosis.

\section{Discussion}

In this study, a defect of renal tubular function in lupus nephritis was mainly reflected by the impaired urinary acidification. We found that urine ammonia was inversely correlated with SLEDAI-2 K scores, rSLEDAI scores, serum creatinine levels and proteinuria, while it was positively correlated with eGFR. And urine titratable acid was only inversely correlated with rSLEDAI scores and proteinuria. However, the strong association between titratable acid excretion and proteinuria is significant even after adjusting for the chronicity index and eGFR at biopsy. Furthermore, urine ammonia was associated with pathological features of chronicity and tubulointerstitial disease activity. Urine ammonia was a potential reflector of disease activity and tubulointerstitial lesions in lupus nephritis.

Lupus nephritis encompasses diverse patterns of renal disease, including glomerular, tubulointerstitial, and vascular lesions [26]. Growing evidence showed that tubulointerstitial lesions might reflect the severity of lupus nephritis [18, 27, 28]. Y Nozaki et al. found that urinary kidney injury molecule-1 (KIM-1), a specific biomarker for acute tubular damage, was increased in LN and correlated with proteinuria [23]. Several studies have indicated that elevated urinary neutrophil gelatinase-associated lipocalin (NGAL) and monocyte chemoattractant protein-1 (MCP-1) in LN were associated with renal injury indices such as serum creatinine and proteinuria [29-31]. These findings were consistent with our study that urine ammonia was correlated with serum creatinine, eGFR, and proteinuria. This phenomenon can be explained by the assumption that glomerular proteinuria damages tubules, leading to interstitial inflammation and fibrosis [28]. Our results were also consistent with the view that tubular damage was correlated with the abatement of GFR [32]. Moreover, urine ammonia levels were found to be negatively correlated with both SLEDAI-2 K scores and rSLEDAI scores. Similarly, Turnier et al. found that urinary S100A4 levels were elevated in patients with active LN, and levels of urine S100A4 decreased upon disease activity improvement [33]. Another study identified that urinary colony-stimulating factor- 1 levels were associated with disease activity, which was a potential biomarker to reflect the onset, recurrence and disease activity of lupus nephritis [27]. These findings supported that urinary biomarkers could serve as sensitive indicators for disease activity in lupus nephritis.

The renal biopsy remains the gold standard for the diagnosis of $\mathrm{LN}$, which has a critical role in guiding therapeutic strategy and predicting prognosis. Y Ding et al. illustrated that urine KIM-1, NGAL, and MCP-1 were sensitive factors for the indication of tubulointerstitial lesions in lupus nephritis, and the combination of NGAL and KIM-1 was identified as a renal prognostic factor [29]. In our study, urine ammonia was negatively correlated with interstitial inflammatory cell infiltration, tubular atrophy, and interstitial fibrosis. And there was a significantly lower trend of urine ammonia in patients with more severe tubulointerstitial lesions. Therefore, our study provided clinical evidence in 
Table 2 Associations of urinary acidification function with clinical and renal pathological features of lupus nephritis ( $n=103)$

\begin{tabular}{|c|c|c|c|}
\hline & $\mathrm{pH}$ & Titratable acid $(\mathrm{mmol} / \mathrm{L})$ & Ammonia (mmol/L) \\
\hline \multicolumn{4}{|l|}{ Clinical data } \\
\hline \multicolumn{4}{|l|}{ Gender } \\
\hline Male & $6.15 \pm 0.71$ & $13(8,16.75)$ & $17(11,26.25)$ \\
\hline Female & $6.11 \pm 0.59$ & $14(8.5,18)$ & $27(16.5,40)$ \\
\hline$p$ & 0.838 & 0.485 & 0.015 \\
\hline \multicolumn{4}{|c|}{ Age (years) } \\
\hline$<30$ & $6.01 \pm 0.61$ & $16(9,23)$ & $27(18,38)$ \\
\hline $30-60$ & $6.19 \pm 0.61$ & $13(8.5,15.5)$ & $26(16,39)$ \\
\hline$>60$ & $6.10 \pm 0.61$ & $8(5.5,15.5)$ & $14(10,28)$ \\
\hline$p$ & 0.385 & 0.050 & 0.086 \\
\hline \multicolumn{4}{|c|}{ Hypertension } \\
\hline Yes & $6.00 \pm 0.60$ & $12.5(9,15.25)$ & $19.5(12.5,31.75)$ \\
\hline No & $6.18 \pm 0.61$ & $14(8,19)$ & $32(17,39)$ \\
\hline$p$ & 0.156 & 0.433 & 0.051 \\
\hline \multicolumn{4}{|c|}{ Nephrotic syndrome } \\
\hline Yes & $6.30 \pm 0.67$ & $12.5(7,16)$ & $21.5(13.25,35.75)$ \\
\hline No & $6.00 \pm 0.54$ & $14(10,20)$ & $27(15.5,41)$ \\
\hline$p$ & 0.013 & 0.048 & 0.168 \\
\hline \multicolumn{4}{|c|}{ SLEDAI-2 K } \\
\hline r & 0.077 & -0.114 & -0.310 \\
\hline$p$ & 0.438 & 0.255 & 0.002 \\
\hline \multicolumn{4}{|l|}{ rSLEDAI } \\
\hline r & 0.216 & -0.374 & -0.445 \\
\hline$p$ & 0.029 & $<0.001$ & $<0.001$ \\
\hline \multicolumn{4}{|c|}{ Laboratory data } \\
\hline \multicolumn{4}{|l|}{ Hematuria } \\
\hline Yes & $6.14 \pm 0.58$ & $14(8,17)$ & $24(14.5,36.5)$ \\
\hline No & $6.09 \pm 0.65$ & $13.5(8.75,21.5)$ & $25.5(15.75,41)$ \\
\hline$p$ & 0.694 & 0.584 & 0.461 \\
\hline \multicolumn{4}{|c|}{ Hypokalemia } \\
\hline Yes & $6.21 \pm 0.58$ & $12.5(7.5,15)$ & $27(14.25,36)$ \\
\hline No & $6.10 \pm 0.62$ & $14(8,19)$ & $24(15.5,38.5)$ \\
\hline$p$ & 0.513 & 0.420 & 0.802 \\
\hline \multicolumn{4}{|c|}{$\mathrm{SCr}(\mathrm{umol} / \mathrm{L})$} \\
\hline r & -0.256 & -0.106 & -0.500 \\
\hline$p$ & 0.009 & 0.293 & $<0.001$ \\
\hline \multicolumn{4}{|c|}{$\mathrm{eGFR}\left(\mathrm{ml} / \mathrm{min} / 1.73 \mathrm{~m}^{2}\right)$} \\
\hline$r$ & 0.240 & 0.107 & 0.438 \\
\hline$p$ & 0.015 & 0.288 & $<0.001$ \\
\hline \multicolumn{4}{|c|}{ Urine protein ( $\mathrm{g} / 24 \mathrm{~h})$} \\
\hline r & 0.226 & -0.301 & -0.230 \\
\hline$p$ & 0.022 & 0.002 & 0.021 \\
\hline \multicolumn{4}{|l|}{$C 3(g / L)$} \\
\hline r & 0.020 & -0.112 & -0.125 \\
\hline$p$ & 0.848 & 0.276 & 0.224 \\
\hline
\end{tabular}


Table 2 Associations of urinary acidification function with clinical and renal pathological features of lupus nephritis ( $n=103)$ (Continued)

\begin{tabular}{|c|c|c|c|}
\hline & $\mathrm{pH}$ & Titratable acid (mmol/L) & Ammonia (mmol/L) \\
\hline \multicolumn{4}{|c|}{ Anti-dsDNA antibody drops } \\
\hline r & -0.146 & 0.142 & 0.068 \\
\hline$p$ & 0.369 & 0.381 & 0.675 \\
\hline \multicolumn{4}{|c|}{ Nephrotic range proteinuria (> $3.5 \mathrm{~g} / 24 \mathrm{~h}$ ) } \\
\hline Yes & $6.28 \pm 0.66$ & $12(7,16)$ & $21.5(13.25,35.75)$ \\
\hline No & $5.99 \pm 0.54$ & $15(11,21.5)$ & $27(15.5,41.5)$ \\
\hline$p$ & 0.014 & 0.022 & 0.029 \\
\hline \multicolumn{4}{|c|}{ Pathological features } \\
\hline \multicolumn{4}{|c|}{ Al scores } \\
\hline r & -0.191 & -0.005 & -0.212 \\
\hline$p$ & 0.054 & 0.961 & 0.033 \\
\hline \multicolumn{4}{|c|}{ Endocapillary hypercellularity } \\
\hline r & -0.170 & 0.146 & -0.027 \\
\hline$p$ & 0.087 & 0.146 & 0.790 \\
\hline \multicolumn{4}{|c|}{ Cellular crescents } \\
\hline r & -0.133 & 0.058 & -0.039 \\
\hline$p$ & 0.182 & 0.563 & 0.698 \\
\hline \multicolumn{4}{|c|}{ Karyorrhexis/fibrinoid necrosis } \\
\hline r & 0.028 & -0.136 & -0.083 \\
\hline$p$ & 0.778 & 0.176 & 0.411 \\
\hline \multicolumn{4}{|c|}{ Subendothelial hyaline deposits } \\
\hline r & -0.075 & -0.001 & -0.090 \\
\hline$p$ & 0.449 & 0.995 & 0.372 \\
\hline \multicolumn{4}{|c|}{ Interstitial inflammatory cell infiltration } \\
\hline r & -0.106 & -0.181 & -0.377 \\
\hline$p$ & 0.288 & 0.070 & $<0.001$ \\
\hline \multicolumn{4}{|c|}{ Glomerular leukocyte infiltration } \\
\hline r & -0.172 & 0.223 & -0.008 \\
\hline$p$ & 0.082 & 0.025 & 0.940 \\
\hline \multicolumn{4}{|c|}{$\mathrm{Cl}$ scores } \\
\hline r & 0.167 & -0.418 & -0.474 \\
\hline$p$ & 0.091 & $<0.001$ & $<0.001$ \\
\hline \multicolumn{4}{|c|}{ Glomerular sclerosis } \\
\hline r & 0.061 & -0.236 & -0.345 \\
\hline$p$ & 0.543 & 0.017 & $<0.001$ \\
\hline \multicolumn{4}{|c|}{ Fibrous crescents } \\
\hline r & -0.107 & -0.072 & -0.222 \\
\hline$p$ & 0.280 & 0.476 & 0.025 \\
\hline \multicolumn{4}{|c|}{ Tubular atrophy } \\
\hline r & 0.258 & -0.447 & -0.457 \\
\hline$p$ & 0.009 & $<0.001$ & $<0.001$ \\
\hline \multicolumn{4}{|c|}{ Interstitial fibrosis } \\
\hline r & 0.166 & -0.421 & -0.419 \\
\hline$p$ & 0.095 & $<0.001$ & $<0.001$ \\
\hline
\end{tabular}




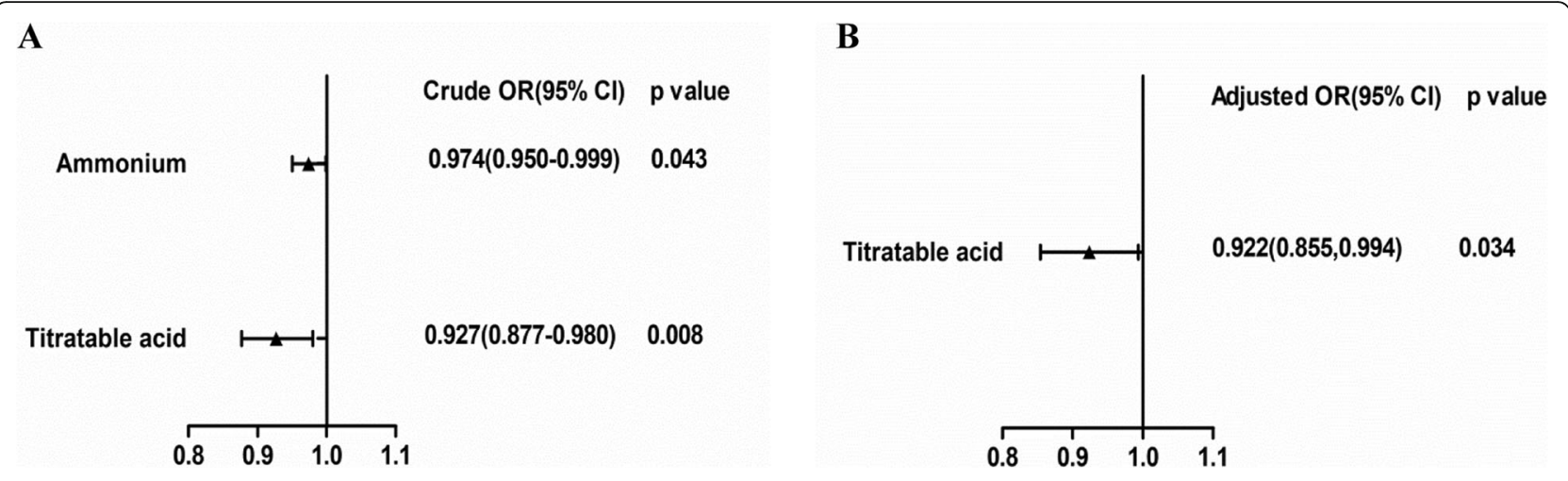

Fig. 1 Logistic regression analysis of factors for distinguishing nephrotic range proteinuria. a. Titratable acid and ammonium were performed in a univariate logistic regression analysis. b. After the adjustment for chronicity index and eGFR, titratable acid was performed in a multivariate logistic regression analysis. Confidence intervals that do not cross the line of identity (1.0) are considered statistically significant. CI confidence interval; OR odds ratio

support of the prior findings that several urinary biomarkers might accurately identity tubulointerstitial damage in LN. Furthermore, active pathological lesions, reflected by cellular crescents and fibrinoid necrosis, foretell adverse disease progression. And chronic lesions also have a high association with disease progression among patients with lupus nephritis [25]. It has been demonstrated that chronic lesions were better than active lesions in reflecting the progressive eGFR decline [3]. In the present study, urine ammonia was negatively correlated with both $\mathrm{AI}$ and $\mathrm{CI}$ scores, especially several indicators of chronic lesions.

Chronic kidney disease patients with clinically normal acid-base status might have acid-related kidney injury because of enhanced ammonia production by per nephron, which promoting tubulointerstitial fibrosis and further kidney disease progression through intrarenal activation of the alternative pathway of complement [34]. In a cohort study, low urine ammonia excretion was associated with an increased risk of CKD progression [14]. Further studies are needed to explore whether urine ammonia is an influencing factor for lupus nephritis progression.

There are several limitations to the present study. First, this study included a small number of patients. Larger cohorts will be needed to confirm our findings. Second, our study focused on relationships between these urinary markers and lupus nephritis clinical and histopathological features, further studies are required to assess potential effects of urine acidification indicators in recurrence, disease progression and prognosis in lupus nephritis. Third, only some patients in our study underwent the measurements of arterial blood gas tests, renal tubular acidosis in this cohort remained unclear.

\section{Conclusion}

Urine ammonia was associated with clinical and pathological features of chronicity and tubulointerstitial disease activity among patients with lupus nephritis. Furthermore, the strong association between urinary protein and titratable acid excretion at the time of kidney biopsy is significant even after adjusting for the chronicity index and eGFR at biopsy. Future studies can further assess serial measures of urine acidification to determine robustness over time.

\section{Abbreviations \\ LN: Lupus nephritis; SLE: Systemic lupus erythematosus; ESRD: End-stage kidney disease; RTA: Renal tubular acidosis; CKD: Chronic kidney disease; GFR: Glomerular filtration rate; BUN: Blood urea nitrogen; C3: Complement 3; ANA: Antinuclear antibodies; anti-dsDNA: Anti-double-stranded DNA; CKD- EPI: Chronic kidney disease epidemiology collaboration; SLEDAI-2 K: SLE disease activity index 2000; rSLEDAl: SLE disease activity index; Al: Activity index; Cl: Chronicity index; KIM-1: Kidney injury molecule-1; NGAL: Neutrophil gelatinase-associated lipocalin; MCP-1: Monocyte chemoattractant protein-1}

\section{Acknowledgments}

The authors thank the patients for participation in our study and the staff of the Department of Nephrology at the First Affiliated Hospital of Nanjing Medical University for assistance.

\section{Fundings}

This work was supported by grants from the National Natural Science Foundation of China (No. 81670628, 81,870,469, 81,300,573), the Natural Science Foundation of Jiangsu Province (No. BK20131030), the "333 Project" of Jiangsu Province, the Six Talent Peaks Project in Jiangsu Province (WSN010), the China Scholarship Council (CSC, File No. 201608320124), Chinese Society of Nephrology (17010060675), and the Priority Academic Program Development (PAPD) of Jiangsu Higher Education Institution. These funding sources were not involved in the design of the study and data collection, analysis, or interpretation. They also played no role in the writing of the manuscript.

\section{Authors' contributions}

$Y Y, C X$, and SD designed the research. HZ, HW, SD, LW, BZ, QL, and SL collected, analyzed and interpreted the data. HZ, HW, CZ, and YY wrote the manuscript and had responsibility for its final content. $Y Y$ is the guarantor of this work, has complete access to all the data in the study, and takes ultimate responsibility for the study design and integrity of data analysis. All authors have read the final manuscript and approved the submission.

Availability of data and materials

The datasets analyzed during the current study are available from the corresponding author on reasonable request. 


\section{Ethics approval and consent to participate}

All procedures performed in studies involving human participants were in accordance with the ethical standards of the ethics committee of the First Affiliated Hospital of Nanjing Medical University and with the 1964 Helsinki declaration and its later amendments or comparable ethical standards. We were allowed to access and use the medical records described in this study by the First Affiliated Hospital of Nanjing Medical University. Written informed consent was obtained from all individual participants included in the study.

\section{Consent for publication}

Not applicable.

\section{Competing interests}

The authors declare that they have no competing interests.

Received: 4 June 2020 Accepted: 14 October 2020

Published online: 20 October 2020

\section{References}

1. Davidson A. What is damaging the kidney in lupus nephritis? Nat Rev Rheumatol. 2016;12(3):143-53.

2. Yung S, Chan TM. Molecular and immunological basis of Tubulo-interstitial injury in lupus nephritis: a comprehensive review. Clin Rev Allergy Immunol. 2017:52(2):149-63.

3. Rijnink EC, Teng YKO, Wilhelmus S, Almekinders M, Wolterbeek R, Cransberg K, Bruijn JA, Bajema IM. Clinical and Histopathologic characteristics associated with renal outcomes in lupus nephritis. Clin J Am Soc Nephrol. 2017;12(5):734-43.

4. Mok CC. Biomarkers for lupus nephritis: a critical appraisal. J Biomed Biotechnol. 2010;2010:638413

5. Moroni G, Depetri F, Ponticelli C. Lupus nephritis: when and how often to biopsy and what does it mean? J Autoimmun. 2016;74:27-40.

6. Malvar A, Pirruccio P, Alberton V, Lococo B, Recalde C, Fazini B, Nagaraja H, Indrakanti $\mathrm{D}$, Rovin $\mathrm{BH}$. Histologic versus clinical remission in proliferative lupus nephritis. Nephrol Dial Transplant. 2017;32(8):1338-44.

7. Soliman S, Mohamed FA, Ismail FM, Stanley S, Saxena R, Mohan C. Urine angiostatin and VCAM-1 surpass conventional metrics in predicting elevated renal pathology activity indices in lupus nephritis. Int J Rheum Dis. 2017; 20(11):1714-27.

8. Lee EJ, Kwon OC, Ghang B, Lim DH, Kim DH, Hong S, Lee CK, Yoo B, Kim YG. Immunoglobulin Binding Protein 1 as a Potential Urine Biomarker in Patients with Lupus Nephritis. Int J Mol Sci. 2019;20(10).

9. Arriens C, Wren JD, Munroe ME, Mohan C. Systemic lupus erythematosus biomarkers: the challenging quest. Rheumatology. 2017;56(suppl_1):i32-45.

10. Michaelson JS, Wisniacki N, Burkly LC, Putterman C. Role of TWEAK in lupus nephritis: a bench-to-bedside review. J Autoimmun. 2012;39(3):130-42.

11. Gil-Pena H, Mejia N, Santos F. Renal tubular acidosis. J Pediatr. 2014;164(4): 691-8 e691.

12. Soleimani M, Rastegar A. Pathophysiology of renal tubular acidosis: Core curriculum 2016. Am J Kidney Dis. 2016;68(3):488-98.

13. Santos F, Gil-Pena H, Alvarez-Alvarez S. Renal tubular acidosis. Curr Opin Pediatr. 2017;29(2):206-10.

14. Vallet M, Metzger M, Haymann JP, Flamant M, Gauci C, Thervet E, Boffa JJ, Vrtovsnik F, Froissart M, Stengel B, et al. Urinary ammonia and long-term outcomes in chronic kidney disease. Kidney Int. 2015;88(1):137-45.

15. Scialla JJ, Asplin J, Dobre M, Chang AR, Lash J, Hsu CY, Kallem RR, Hamm LL, Feldman HI, Chen J, et al. Higher net acid excretion is associated with a lower risk of kidney disease progression in patients with diabetes. Kidney Int. 2017:91(1):204-15.

16. Zhu H, Liu X, Zhang C, Li Q, An X, Liu S, Wu L, Zhang B, Yuan Y, Xing C. Association of urinary acidification function with the progression of diabetic kidney disease in patients with type 2 diabetes. J Diabetes Complicat. 2019; 33(11):107419.

17. Broder A, Mowrey WB, Khan HN, Jovanovic B, Londono-Jimenez A, Izmirly P, Putterman C. Tubulointerstitial damage predicts end stage renal disease in lupus nephritis with preserved to moderately impaired renal function: a retrospective cohort study. Semin Arthritis Rheum. 2018;47(4):545-51.

18. Yu F, Wu LH, Tan Y, Li LH, Wang CL, Wang WK, Qu Z, Chen MH, Gao JJ, Li $Z Y$, et al. Tubulointerstitial lesions of patients with lupus nephritis classified by the 2003 International Society of Nephrology and Renal Pathology Society system. Kidney Int. 2010;77(9):820-9.
19. Li SL, Liou LB, Fang JT, Tsai WP. Symptomatic renal tubular acidosis (RTA) in patients with systemic lupus erythematosus: an analysis of six cases with new association of type 4 RTA. Rheumatology. 2005;44(9):1176-80.

20. Petri M, Orbai AM, Alarcon GS, Gordon C, Merrill JT, Fortin PR, Bruce IN, Isenberg D, Wallace DJ, Nived O, et al. Derivation and validation of the systemic lupus international collaborating clinics classification criteria for systemic lupus erythematosus. Arthritis Rheum. 2012;64(8):2677-86.

21. Weening JJ, D'Agati VD, Schwartz MM, Seshan SV, Alpers CE, Appel GB, Balow JE, Bruijn JA, Cook T, Ferrario F, et al. The classification of glomerulonephritis in systemic lupus erythematosus revisited. Kidney Int. 2004;65(2):521-30.

22. Levey AS, Stevens LA, Schmid CH, Zhang YL, Castro AF 3rd, Feldman HI, Kusek JW, Eggers P, Van Lente F, Greene T, et al. A new equation to estimate glomerular filtration rate. Ann Intern Med. 2009;150(9):604-12.

23. Nozaki Y, Kinoshita K, Yano T, Shiga T, Hino S, Niki K, Kishimoto K, Funauchi M, Matsumura I. Estimation of kidney injury molecule-1 (Kim-1) in patients with lupus nephritis. Lupus. 2014;23(8):769-77.

24. Hill GS, Delahousse M, Nochy D, Tomkiewicz E, Remy P, Mignon F, Mery JP. A new morphologic index for the evaluation of renal biopsies in lupus nephritis. Kidney Int. 2000;58(3):1160-73.

25. Austin HA 3rd, Muenz LR, Joyce KM, Antonovych TT, Balow JE. Diffuse proliferative lupus nephritis: identification of specific pathologic features affecting renal outcome. Kidney Int. 1984;25(4):689-95.

26. Kaul A, Agrawal V, Bhaduaria D, Agrawal V, Prasad N, Gupta A, Sharma RK. Vasculitis and vasculopathy in lupus nephritis: clinical variability, outcome, and new insight into treatment. Saudi J Kidney Dis Transplant. 2017;28(2):415-24.

27. Menke J, Amann K, Cavagna L, Blettner M, Weinmann A, Schwarting A, Kelley VR. Colony-stimulating factor-1: a potential biomarker for lupus nephritis. J Am Soc Nephrol. 2015;26(2):379-89.

28. Hill GS, Delahousse M, Nochy D, Mandet C, Bariety J. Proteinuria and tubulointerstitial lesions in lupus nephritis. Kidney Int. 2001;60(5):1893-903.

29. Ding Y, Nie LM, Pang Y, Wu WJ, Tan Y, Yu F, Zhao MH. Composite urinary biomarkers to predict pathological tubulointerstitial lesions in lupus nephritis. Lupus. 2018;27(11):1778-89.

30. Zivkovic V, Cvetkovic T, Mitic B, Stamenkovic B, Stojanovic S, Radovanovic-Dinic $B$, Jurisic V. Monocyte chemoattractant protein-1 as a marker of systemic lupus erythematosus: an observational study. Rheumatol Int. 2018;38(6):1003-8.

31. Torres-Salido MT, Cortes-Hernandez J, Vidal X, Pedrosa A, Vilardell-Tarres M, Ordi-Ros J. Neutrophil gelatinase-associated lipocalin as a biomarker for lupus nephritis. Nephrol Dial Transplant. 2014;29(9):1740-9.

32. Waikar SS, Sabbisetti V, Arnlov J, Carlsson AC, Coresh J, Feldman HI, Foster MC, Fufaa GD, Helmersson-Karlqvist J, Hsu CY, et al. Relationship of proximal tubular injury to chronic kidney disease as assessed by urinary kidney injury molecule-1 in five cohort studies. Nephrol Dial Transplant. 2016;31(9):1460-70.

33. Turnier JL, Fall N, Thornton S, Witte D, Bennett MR, Appenzeller S, KleinGitelman MS, Grom AA, Brunner HI. Urine S100 proteins as potential biomarkers of lupus nephritis activity. Arthritis Res Ther. 2017;19(1):242.

34. Nagami GT, Hamm LL. Regulation of Acid-Base balance in chronic kidney disease. Adv Chronic Kidney Dis. 2017;24(5):274-9.

\section{Publisher's Note}

Springer Nature remains neutral with regard to jurisdictional claims in published maps and institutional affiliations.

Ready to submit your research? Choose BMC and benefit from:

- fast, convenient online submission

- thorough peer review by experienced researchers in your field

- rapid publication on acceptance

- support for research data, including large and complex data types

- gold Open Access which fosters wider collaboration and increased citations

- maximum visibility for your research: over $100 \mathrm{M}$ website views per year

At BMC, research is always in progress.

Learn more biomedcentral.com/submissions 